\title{
Finite approximations to the second-order properties closure in single phase polycrystals
}

\author{
Brent L. Adams \\ b_I_adams@byu.edu \\ Xiang Gao \\ Surya R. Kalidindi
}

Follow this and additional works at: https://scholarsarchive.byu.edu/facpub

Part of the Mechanical Engineering Commons

\section{Original Publication Citation}

Acta Materialia 53 (25) 3563-3577

\section{BYU ScholarsArchive Citation}

Adams, Brent L.; Gao, Xiang; and Kalidindi, Surya R., "Finite approximations to the second-order properties closure in single phase polycrystals" (2005). Faculty Publications. 390.

https://scholarsarchive.byu.edu/facpub/390 


\title{
Finite approximations to the second-order properties closure in single phase polycrystals
}

\author{
Brent L. Adams ${ }^{\mathrm{a}, *}$, Xiang (Carl) Gao ${ }^{\text {a }}$, Surya R. Kalidindi ${ }^{\mathrm{b}}$ \\ ${ }^{a}$ Department of Mechanical Engineering, Brigham Young University, Provo, UT 84602, United States \\ ${ }^{\mathrm{b}}$ Department of Materials Science and Engineering, Drexel University, Philadelphia, PA 19104, United States
}

\begin{abstract}
Extension of the first-order theory of microstructure design to considerations of morphological texture is addressed in this paper. The main challenges include the $r$-interdependence of the 2-point correlation functions of lattice orientation, construction of the corresponding microstructure hull, and its corresponding properties closure(s). It is shown that the correlation functions can be expressed in terms of an intermediate construct, called the texture function; the correlation functions have quadratic dependence in the texture functions. A complete (finite) texture hull is readily constructed for the texture functions in Fourier space, and is found to be a convex polytope. Eigen-texture functions occupy its corner (extreme) points. Microstructure design proceeds directly from homogenization relations evaluated at the corner points. This gives rise to (combined) properties closures, from which second-order microstructure design can proceed. This is demonstrated in a brief case study.
\end{abstract}

Keywords: Mesostructure; Microstructure-texture; Statistical mechanics; Mechanical properties-elastic behavior

\section{Introduction}

Within the context of statistical continuum theory, refined bounds and estimates of effective properties require information about microstructure beyond the volume fractions required by the first-order theories. For example, expressions for the fourth-order effective elasticity tensor, $C^{*}$, can be expressed as a geometric series in the correlation functions of local stiffness [1-3]:

$C^{*}=\langle C\rangle-\left\langle C^{\prime} \Gamma C^{\prime}\right\rangle+\left\langle C^{\prime} \Gamma C^{\prime} \Gamma C^{\prime}\right\rangle-\cdots$

Here, the angular brackets, $\langle\cdot\rangle$, denote ensemble averages, $C^{\prime}=C-C^{\mathrm{r}}$ is the polarization of local stiffness $C$ with respect to a selected reference stiffness, $C^{\mathrm{r}}$, and $\Gamma$ denotes an appropriate Green's function operator associated with solutions to the basic governing equa-

\footnotetext{
* Corresponding author. Tel.: +1 801422 7124; fax: +1 8014220516 . E-mail address: B_L_Adams@byu.edu (B.L. Adams).
}

tions for static linear elastic properties subject to homogeneous boundary conditions. The basic feature of Eq. (1) is a hierarchical dependence upon an ascending order of correlation functions; thus, the first-order term requires $\langle C(x)\rangle$, the second-order term $\left\langle C^{\prime}(x) C^{\prime}\left(x^{\prime}\right)\right\rangle$, and so forth. This hierarchy is found in other theories, such as the solution to the Navier-Stokes relations for the visco-plastic modulus $[4,5]$.

\subsection{First-order theory for microstructure design}

First-order effective properties theories, of the type mentioned, have recently been incorporated in a new methodology for tailoring the microstructure of materials to meet the requirements of mechanical design [6-9]. The goal of microstructure design is to begin with the combination of properties of importance to a specific design problem, and then to derive the class of microstructures that are predicted to best satisfy them. The new 
work builds upon the methodology of Bunge [10], which describes the orientation distribution function (ODF), $f(g)$, in terms of an infinite Fourier series,

$f(g)=\sum_{l=0}^{\infty} \sum_{\mu=1}^{M(l)} \sum_{v=1}^{N(l)} C_{l}^{\mu v} \dot{T}_{l}^{\mu v}(g)$

Here, $g$ denotes the orientation of the lattice of any particular crystallite, with respect to a reference crystallite, and $\dddot{T}_{l}^{\mu \nu}(g)$ are the generalized spherical harmonic functions, symmetrized according to the symmetry subgroups of the lattice and the processing, as may be appropriate. The set of Fourier coefficients, $\left\{C_{l}^{\mu \nu}\right\}$, define a point in the infinite dimensional Fourier space, representing the ODF. The set of all possible ODFs is called the hull of ODFs, as it is found to be a convex set, compact in all of its sub-dimensions [6]. It was also shown that the effective properties (homogenization) relations themselves define hyper-planes or surfaces in the Fourier space, delineating ODFs within the hull that are predicted to achieve specified properties. Later work demonstrated that ordinary methods of linear analysis recover the set of properties combinations predicted by the associated homogenization relations [7]. This set is called the properties closure.

Properties closure is closely related to the important G-closure problem for linear properties, which is defined to be the set of all possible effective properties of all possible composites assembled from a given set of phases $[11,12]$. Given that the properties closures of the present work are estimated from homogenization relations of finite order, they can be considered to be approximations of G-closure.

One key challenge in searching the microstructure hull is to insure that a given ODF, represented by its Fourier coefficients, actually lies in the physically allowed hull of ODFs. This was treated in a particular way, using Gramm-Schmidt orthogonalization [9].

Common design problems treated by the methodology using first-order homogenization theories include elasticplastic treatment of compliant beams [6] and the loadbearing capacity of plates containing holes [7-9].

The work to date has shown a remarkable degree of invertibility in the homogenization relations. Although much of modern thinking in materials design follows exclusively the traditional 'forward direction' of materials science and engineering [viz. processing $\Rightarrow$ microstructure $\Rightarrow$ properties], forward design has been limited to considerations of only a tiny fraction of the space of all microstructures. By contrast, the new methodology is able to precisely define the complete set (hull) of all microstructures; and since the homogenization relations themselves are described as sets that intersect the hull, an invertible mapping of the form [properties $\Rightarrow$ microstructure] is obtained. The current weakness of the new methodology is that it does not yet adequately consider processing constraints and thermodynamic stability. For this reason it is presently considered to be a tool for preliminary or exploratory design.

\subsection{Extending microstructure design theory to second-order}

The extent to which invertibility can be achieved for other, more advanced homogenization relations and concomitant microstructure representations remains to be explored. In this paper, we address mainly the problem of constructing the hull for 2-point orientation correlation functions (OCFs), as required, for example, by the second term in the homogenization relation given by relation (1). It is known that homogenization relations based only upon volume-fraction information may be less than adequate to conduct useful design. Elastic bound estimates for polycrystals, for example, are often separated by $\sim 10 \%$ or more of the estimate itself [13], and this may be unacceptable in highly constrained design. Remedy by resort to higher-order homogenization relations is possible if we are able to construct an appropriate hull for the 2-point correlation functions. This possibility was mentioned in the earliest work describing the new methodology [6], but the complexities of the 2-point representations were not fully appreciated.

The principal challenge associated with the 2-point functions is a complex interdependency in the $r$-variable of the representation itself. Consider the joint probability density that the tail and head of a vector, $r$, randomly placed in the microstructure of a single phase polycrystal, associates with lattice orientations $g$ and $g^{\prime}$, respectively. In statistically homogeneous microstructures, this probability density is labeled $f_{2}\left(g, g^{\prime} \mid r\right)$; it is also called the 2-point OCF, or the OCF. Modern methods of automated electron backscattering diffraction enable statistically reliable estimates of this function to be obtained by experimental sampling of the microstructure [13]. A limited understanding of the $r$-interdependence of the OCF can be seen in the following conservation relationship that must hold in statistically homogeneous microstructures:

$\iiint_{\Psi(\Omega)} f_{2}\left(g, g^{\prime} \mid r\right) \theta(r) \mathrm{d} r=f(g) f\left(g^{\prime}\right)$.

Here, $\Psi(\Omega)$ designates the complete set of all possible vectors $r$ that can be found in any particular region of 3 -D space, $\Omega$. When pairs of points are introduced into random and independent locations within $\Omega, \theta(r)$ is the geometrical probability density for the occurrence of vector $r$ among these pairs. It is evident that relation (3) defines a necessary system of constraints, linking the OCFs to one-another. However, the question that 
arises is whether these constraints are sufficient to insure that a set of OCFs is physically realizable in the ensemble. Torquato [14] reviewed what is known about the related problem of physical realizability for the autocovariance function in 2-phase composites, and concluded that the problem remains an open and unsolved one. Addressing the $r$-interdependence of the OCF is the chief obstacle to a formulation of microstructure design at the second-order level.

\subsection{Organization of the paper}

The purpose of this paper is to describe an approximate method for obtaining the hull of OCFs - a method that overcomes the problem of $r$-interdependence. An essential intermediate step is the quantitative representation of the microstructures of samples belonging to a statistical ensemble. Discrete, piecewise continuous representations are introduced by partitioning the homogeneous space of orientations into a finite set of sub-spaces. Spatial attributes of sample microstructures are obtained in like manner by partitioning sample region $\Omega$ into a finite number of sub-regions. This is described in Section 2. In Section 3, the fundamental equation, linking the 2-point OCFs in each sample to the spatial coordination of orientations found among the sub-cells, is presented. These local OCFs are readily formed by integration, and passage to the ensemble relates them to the overall, global OCFs describing the microstructure of the statistical ensemble. The advantage of forming the OCFs in this manner is that the problems of $r$-interdependency are overcome. Section 4 describes construction of the hull of texture functions, which is found to be more convenient for computations than the hull for OCFs (which resides in a space of much higher dimension). Section 5 describes a transformation of basis from the indicator functions to the classical generalized spherical harmonic functions for orientation variables, and to the Haar wavelet functions for the spatial variables. These alternate bases can facilitate a deeper physical understanding of microstructure and homogenization relations. In Section 6 we relate the global and local 2-point OCFs to the elastic properties using relation (1). Basic strategy for conducting microstructure design is outlined in Section 7, and illustrated with a brief example extending microstructure design methodology to second-order for a particular optimized ODF obtained by first-order microstructure design [9].

\subsection{Notational conventions}

In the work that follows need will arise for the use of many indices to enumerate orientational and spatial basis functions and their coefficients. It will be useful to contract the number of indices needed into a single index representing an entire set of related indices. Also, we adopt the summation convention, viz. that repeated indices indicate summation over that index, when the repetition occurs on one side of the equation. Exceptions to this rule will be clearly noted. For example, relation (2) will be expressed in abbreviated form as

$f(g)=C^{L} \dot{T}^{L}(g)$,

where the single index $L$ implies all possible $\{l, \mu, n\}$ required to enumerate the orthonormal subspaces of orientation space. Given that $L$ is repeated on the right hand side, summation is implied. We shall use the convention that upper indices are associated with orientation variables and lower indices with spatial ones.

Also, since there is little possibility for confusion, we will omit reference to any particular sample of the ensemble. For example, we use the symbol $\Omega$ to mean the space occupied by any particular sample of the ensemble, rather than the full notation ${ }^{(k)} \Omega$ for the $k$ th element of the ensemble, except where the index is required for clarity.

\section{Representation of microstructure in rectangular regions}

For purposes of pedagogical clarity we restrict our considerations to polycrystalline materials comprising a single phase; but extensions to polyphase polycrystalline materials would be straightforward. Let $M(x, g)$ represent the meso-scale (i.e., granular) microstructure of a sample that occupies region $\Omega$ of 3-D space. $M(x, g)$ is a real valued function on the product space $\Omega \times \mathrm{FZ}$, where 'FZ' denotes the fundamental zone of all physically distinct orientations of the lattice of the crystalline phase. (Formally, $\mathrm{FZ}=\mathrm{SO}(3) / G$, where $G$ is the symmetry subgroup of the crystal lattice, and $\mathrm{SO}(3)$ is the special orthogonal group of rotations in 3-dimensional space. See Morawiec [15] for a full treatment of the FZ for all crystal classes.) Hereafter we refer to $M(x, g)$ as an example of a texture function (TF) associated with a specified sample with microstructure occupying region $\Omega$, as it carries information on both the orientational and morphological textures of the sample. The sample need not be a representative volume element in the development that follows. Formally, $M(x, g) \mathrm{d} g$ is defined to be the volume fraction of material $\mathrm{d} V / V$ in an infinitesimal neighborhood of material point $x$ that associates with lattice orientation lying within an infinitesimal neighborhood of (invariant) measure $\mathrm{d} g$ of the specified orientation $g$ :

$M(x, g) \mathrm{d} g=\mathrm{d} V / V$.

For those familiar with the ODF, for any specified position $x$, the TF can be thought of as specifying the local ODF in the neighborhood of $x$. Functions similar to the $\mathrm{TF}$ have been introduced elsewhere in the literature $[16,17]$. 
If the TF is integrated over its spatial variables, the result must be the ODF in the sample occupying region $\Omega$ :

$\frac{1}{\operatorname{vol}(\Omega)} \iiint_{x \in \Omega} M(x, g) \mathrm{d} x=f(g)$.

Further, when integrated over the orientation variables, the following normalization is taken to hold, consistent with established convention for the ODF [10]:

$\iiint_{g \in \mathrm{FZ}} M(x, g) \mathrm{d} g=1 \quad($ for all $x)$.

Note that the usual parameterization of orientation $g$ is in terms of Euler-angles, with an associated invariant measure of $\mathrm{d} g=\frac{\mathrm{O}(G)}{8 \pi^{2}} \sin \Phi \mathrm{d} \Phi \mathrm{d} \varphi_{1} \mathrm{~d} \varphi_{2}$. Here, $\varphi_{1}, \Phi, \varphi_{2}$ are the Euler angles according to the Bunge convention [10], and $\mathrm{O}(G)$ denotes the order or number of elements belonging to symmetry subgroup $G$ of the FZ.

The TF, although limited in its scope of microstructure representation, is a very complex function, and unsuited for routine calculations. More accessible approximations endowed with limited spatial and orientational resolution are considered here. Approximation of the TF based upon Fourier representation by indicator functions is convenient to obtain the main results presented in this paper. We hereafter refer to the representation of the TF by indicator functions as the primitive representation, and the basis of indicator functions as the primitive basis. Transformation of the primitive basis to another comprising classical generalized spherical harmonic functions for the orientation variables, and Haar wavelets for the spatial variables, is also useful for its physical clarity, and will be presented in Section 5.

\subsection{Representation of TF by indicator functions}

Consider rectangular samples of size $D_{1} \times D_{2} \times D_{3}$. Thus, the real interval $\left[0, D_{k}\right)$ represents the range of possible values for spatial variables $x_{k}(k=1,2,3)$. With an eye towards compatibility with later representations by wavelet functions, $D_{k}$ are selected such that they accept partitioning into $2^{P_{k}}$ sub-intervals of fixed size $\delta=D_{k} / 2^{P_{k}}$. Let the positive integers $s_{k}, 1 \leqslant s_{k} \leqslant 2^{P_{k}}$, enumerate the cubical sub-cells of the sample, each occupying region $\omega_{s_{1} s_{2} s_{3}} \subset \Omega$. These sub-cells must satisfy the properties

$\omega_{s} \cap \omega_{s^{\prime}}=\varnothing\left(s \neq s^{\prime}\right), \quad \bigcup_{s=1}^{s} \omega_{s}=\Omega$.

The explicit definition of the sub-cells is

$$
\begin{aligned}
\omega_{s_{1} s_{2} s_{3}}= & \left\{\left(x_{1}, x_{2}, x_{3}\right) \mid\left(s_{1}-1\right) \delta \leqslant x_{1}<s_{1} \delta,\right. \\
& \left.\left(s_{2}-1\right) \delta \leqslant x_{2}<s_{2} \delta,\left(s_{3}-1\right) \delta \leqslant x_{3}<s_{3} \delta\right\} .
\end{aligned}
$$

Invoking the index contraction convention, the sub-cells will be identified by a single index $s: \omega_{s_{1} s_{2} s_{3}} \leftrightarrow \omega_{s}$. The number of indices involved is $S=2^{\left(P_{1}+P_{2}+P_{3}\right)}$.
Spatial indicator functions can be defined for each sub-cell. These are

$\chi_{s}(x)= \begin{cases}1 & \text { if } x \in \omega_{s} \\ 0 & \text { otherwise }\end{cases}$

Note the compact support for the indicator functions, each in its specified region. Also note that the spatial resolution of the indicator basis is just $\left(\delta^{3} / D_{1} D_{2} D_{3}\right)=1 / S$. Also, note that the spatial indicator functions satisfy the following orthogonality conditions

$\frac{1}{\delta^{3}} \iiint_{\Omega} \chi_{s}(x) \chi_{s^{\prime}}(x) \mathrm{d} x=\delta_{s s^{\prime}}$,

where $\delta_{s s^{\prime}}$ is the Kronecker delta, which equals 1 when $s=s^{\prime}, 0$ otherwise.

Similarly, the FZ of orientations can be partitioned into $N$ sub-regions, say $\gamma_{n}$, each containing a particular orientation $g_{n}$ at the centroid. These sub-regions are selected to have the following properties:

$$
\begin{array}{r}
\gamma_{n} \cap \gamma_{m}=\varnothing(n \neq m), \quad \bigcup_{n=1}^{N} \gamma_{n}=F Z, \\
\iiint_{\gamma_{n}} \mathrm{~d} g=1 / N \quad(\text { for all } n) .
\end{array}
$$

The last relation in (12) is taken for convenience; it means that the orientation sub-regions each have the same measure. Note that $1 / N$ specifies the resolution of orientation that we seek in partitioning the FZ. Associated with each sub-region, define an indicator function $\chi^{n}(g)$ such that

$\chi^{n}(g)= \begin{cases}1 & \text { if } g \in \gamma_{n}, \\ 0 & \text { otherwise. }\end{cases}$

These also satisfy orthogonality conditions

$N \iiint_{\mathrm{FZ}} \chi^{n}(g) \chi^{n^{\prime}}(g) \mathrm{d} g=\delta^{n n^{\prime}}$.

The TF accepts a primitive Fourier approximation in the product space of indicator functions:

$M(x, g) \approx D_{s}^{n} \chi_{s}(x) \chi^{n}(g)$.

Representing microstructure using the primitive indicator bases is similar to modeling a microstructure with the cubical sub-cells representing the constituent grains. However, the definition used here is broader, since each sub-cell may have a distribution comprising more than one orientation. The present definition is consistent with and motivated by experimental considerations. When microscopy is conducted near the limiting spatial resolution of the instrument, volume fractions of the local orientation in the information volume can often be estimated, but the details of how the distribution of orientation is distributed spatially is lacking. The present definition enables this limiting spatial resolution to be incorporated in the representation directly, without giving up the local distribution of orientation. 
Equality in relation (15) is achieved only in the limit that $S$ and $N$ both go to infinity. Extending relation (6) to considerations of individual cubical sub-cells, one can describe the local ODF for a single sub-cell of the sample, ${ }_{s} f(g)$ :

$$
\begin{aligned}
\frac{1}{\delta^{3}} & \iiint_{x \in \Omega} M(x, g) \chi_{s}(x) \mathrm{d} x \\
& =\frac{1}{\delta^{3}} \iiint_{x \in \omega_{s}} M(x, g) \mathrm{d} x={ }_{s} f(g)=D_{s}^{n} \chi^{n}(g) .
\end{aligned}
$$

When combined with the normalization relation (7), one finds the following normalizations among the Fourier coefficients of the primitive basis:

$\sum_{s=1}^{S} \sum_{n=1}^{N} D_{s}^{n}=S N$

and

$\sum_{n=1}^{N} D_{s}^{n}=N \quad($ for all $s)$.

Also, given the definition of the TF (5), the definition of its primitive Fourier approximation (15), and normalization condition (18), it is readily seen that the texture coefficients are bounded:

$0 \leqslant D_{s}^{n} \leqslant N$.

If sub-cell $s^{*}$ contains only one orientation, say $g^{*} \in \gamma_{n^{*}}$, then $D_{s^{*}}^{n^{*}}=N$ and all other $D_{s^{*}}^{n \neq n^{*}}=0$. If more than one orientation is found in sub-cell $s^{*}$, the Fourier coefficients will be less than $N$, but greater than 0 , and they will sum to $N$ according to (18). Relations (17)-(19) help establish the hull of TFs, and will be considered again in Section 4.

\subsection{Relationships among the localized and global texture coefficients}

The global TF is related to the ensemble average of the local TFs of the samples:

$\langle M(x, g)\rangle \approx\left\langle D_{s}^{n}\right\rangle \chi_{s}(x) \chi^{n}(g)$,

where the ensemble average is defined by

$\langle(\cdot)\rangle=\frac{1}{K} \sum_{k=1}^{K}{ }^{(k)}(\cdot)$,

with $K$ denoting the number of elements within the ensemble.

\section{2-Point spatial correlations of orientation}

Next, the construction of the 2-point OCF is undertaken, using the TF of the sample as an intermediate construct. The advantage is that we are assured that the OCFs so obtained will be physically realizable. Since $M(x, g)$ is interpreted to be the volume-fraction density of crystalline material of orientation $g$ in the near vicinity of point $x$ in region $\Omega$, then the joint density of material of orientation $g$ at point $x$ and of orientation $g^{\prime}$ at position $x^{\prime}$ is the product $M(x, g) M\left(x^{\prime}, g^{\prime}\right)$. When a single element of the ensemble is under consideration, this joint density is of limited importance. However, the ensemble average of this product, $\left\langle M(x, g) M\left(x^{\prime}, g^{\prime}\right)\right\rangle$, carries the useful notion of the statistical occurrence of pairs of orientations of various types, at the specified positions, in the ensemble. It follows that in the ensemble of microstructures, the difference $\left\langle M(x, g) M\left(x^{\prime}, g^{\prime}\right)\right\rangle$ $-f(g) f\left(g^{\prime}\right)$ describes the polarization of coherence or polarization of correlation of the specified pair of orientations at the specified positions. If this difference is positive, there is an attraction between the orientations, beyond that which can be explained by random placement of volume fractions alone, which is the product $f(g) f\left(g^{\prime}\right)$. If negative, there is repulsion. These correlations are presumed to occur because of the complex details of microstructure formation and transformation during the manufacture of the material. They constitute the meso-scale morphological texture of the polycrystal.

If $\left\langle M(x, g) M\left(x+r, g^{\prime}\right)\right\rangle$, is independent of position $x$ $\left(r=x^{\prime}-x\right)$, the microstructure of the ensemble is said to possess statistical homogeneity. Most representations of microstructure and effective microstructure/properties relations assume statistical homogeneity, if they must depend upon ordinary experimental measurements. Opacity of most polycrystalline materials to X-ray and electron probes effectively limits experimental measurements to the ensemble rather than individual samples. We note, however, that recent work with high-energy X-rays [18] or automated serial sectioning [19] may reduce this limitation in the future. In the sequel we shall assume that the microstructure of the ensemble is statistically homogeneous.

\subsection{The fundamental relation}

Define the local OCF at separation $r$ to be $f_{2}\left(g, g^{\prime} \mid r\right)$ :

$\frac{1}{\operatorname{vol}(\Omega \mid r)} \iiint_{x \in \Omega \mid r} M(x, g) M\left(x+r, g^{\prime}\right) \mathrm{d} x=f_{2}\left(g, g^{\prime} \mid r\right)$,

where $\Omega \mid r \subset \Omega$ symbolizes the set of those points $x$ lying within $\Omega$ for which, also, $(x+r) \in \Omega$. Relation (22) is the fundamental relation linking the TFs of selected samples to their local OCFs. Physically, $f_{2}\left(g, g^{\prime} \mid r\right)$ describes the expectation for joint volume density for orientation $g$ at the tail and orientation $g^{\prime}$ at the head for randomly placed vector $r$, falling entirely in $\Omega$. Thus, assuming that all samples of the ensemble occupy a region of 
the same size and shape, $\Omega$, the ensemble OCF is $\left\langle f_{2}\left(g, g^{\prime} \mid r\right)\right\rangle$. As previously mentioned, it is the ensemble OCF that is relevant to typical homogenization relationships of statistical continuum theory. The main idea taken here is to represent the ensemble OCF, approximately, using a Fourier series comprising products of spatial and orientational indicator functions, and to relate the ensemble OCF to the local OCFs of the samples from which it is constructed.

\subsection{Representation and evaluation of the fundamental relation in Fourier space}

The reader will note that the range of the vector $r$ is over all possible pairs of points lying in $\Omega$. But this region is also rectangular, and exactly twice the size of $\Omega$ in each of its dimensions, or 8 times the volume of $\Omega$ in the 3-dimensional real space. Label this set of possible $r$ vectors $\Psi(\Omega)$. It is defined as

$$
\begin{aligned}
\Psi(\Omega)= & \left\{\left(r_{1}, r_{2}, r_{3}\right) \mid-D_{1} \leqslant r_{1}<D_{1},\right. \\
& \left.-D_{2} \leqslant r_{2}<D_{2},-D_{3} \leqslant r_{3}<D_{3}\right\} .
\end{aligned}
$$

This region can also be subdivided into sub-cells, in a manner similar to the partitioning of $\Omega$. Let these subcells be $\psi_{t_{1} t_{2} t_{3}} \leftrightarrow \psi_{t}$, where the indices $t_{1}, t_{2}, t_{3} \leftrightarrow t$ are integers that enumerate in the 3-dimensions, as before. The real interval $\left[-D_{k}, D_{k}\right)$ contains all possible values of the variable $r_{k}$. Partition this interval into $2^{P_{k}^{\prime}}$ subintervals of size $\delta^{\prime}=D_{k} / 2^{\left(P_{k}^{\prime}-1\right)}$. Note that the sub-cell size $\delta^{\prime}$ is not necessarily dictated by $\delta$. However, given the fact that spatial resolution of the approximate representation of sample microstructure is limited to $\delta$, for that which follows we stipulate $\delta^{\prime}=\delta$. It follows that $P_{k}^{\prime}=P_{k}+1$. Consistent with this scheme, the sub-cells themselves are defined by

$$
\begin{aligned}
& \psi_{t} \leftrightarrow \psi_{t_{1} t_{2} t_{3}} \\
& =\left\{\begin{array}{l|l}
\left(r_{1}, r_{2}, r_{3}\right) & \begin{array}{l}
\left(t_{1}-2^{P_{1}^{\prime}-1}-1\right) \delta^{\prime} \leqslant r_{1}<\left(t_{1}-2^{P_{1}^{\prime}-1}\right) \delta^{\prime}, \\
\left(t_{2}-2^{P_{2}^{\prime}-1}-1\right) \delta^{\prime} \leqslant r_{2}<\left(t_{2}-2^{P_{2}^{\prime}-1}\right) \delta^{\prime}, \\
\left(t_{3}-2^{P_{3}^{\prime}-1}-1\right) \delta^{\prime} \leqslant r_{3}<\left(t_{3}-2^{P_{3}^{\prime}-1}\right) \delta^{\prime} ; \\
1 \leqslant t_{k} \leqslant 2^{P_{k}^{\prime}} \text { for } k=1,2,3
\end{array}
\end{array}\right\}
\end{aligned}
$$

indicator functions on these sub-cells $\psi_{t}$ are defined as before

$$
\chi_{t}(r)= \begin{cases}1 & \text { if } r \in \psi_{t} \\ 0 & \text { otherwise }\end{cases}
$$

The local OCF then accepts the following representation using the indicator basis for the $r$-dependence:

$f_{2}\left(g, g^{\prime} \mid r\right) \approx F_{t}^{n n^{\prime}} \chi^{n}(g) \chi^{n^{\prime}}\left(g^{\prime}\right) \chi_{t}(r)$.

Introducing the Fourier representation of the TF (16) into the fundamental equation (22) we obtain

$$
\begin{aligned}
f_{2}\left(g, g^{\prime} \mid r\right) \approx & D_{s}^{n} D_{s^{\prime}}^{n^{\prime}} \chi^{n}(g) \chi^{n^{\prime}}\left(g^{\prime}\right) \\
& \times\left[\frac{1}{\operatorname{vol}(\Omega \mid r)} \oiiint_{\Omega} \chi_{s}(x) \chi_{s^{\prime}}(x+r) \mathrm{d} x\right] .
\end{aligned}
$$

The last term in (27), involving the integration over $\Omega$, carries the $r$-dependence of the local OCF. It is convenient to approximate this function with its own Fourier series, with an indicator-function basis over the sub-cells $\psi_{t}$. Proceeding in this direction,

$$
\begin{aligned}
h_{s s^{\prime}}(r) & =\frac{1}{\operatorname{vol}(\Omega \mid r)} \oiiint_{\Omega} \chi_{s}(x) \chi_{s^{\prime}}(x+r) \mathrm{d} x \\
& \approx H_{s s^{\prime} t^{\prime}} \chi_{t^{\prime}}(r) .
\end{aligned}
$$

Multiplying both sides of this equation by $\chi_{t}(r)$, and integrating over all $r \in \Psi(\Omega)$, we obtain

$$
\begin{aligned}
H_{s s^{\prime} t} & =\oiiint_{\Omega} \chi_{s}(x) \oiiint_{\Psi(\Omega)} \frac{1}{\delta^{\prime 3} \operatorname{vol}(\Omega \mid r)} \chi_{t}(r) \chi_{s^{\prime}}(x+r) \mathrm{d} r \mathrm{~d} x \\
& =\oiiint_{\omega_{s}} \chi_{s}(x) \oiiint_{\psi_{t}} \frac{1}{\delta^{\prime 3} \operatorname{vol}(\Omega \mid r)} \chi_{s^{\prime}}(x+r) \mathrm{d} r \mathrm{~d} x .
\end{aligned}
$$

The sub-volume sampled by vector $r$ is readily expressed for rectangular models as

$\operatorname{vol}(\Omega \mid r)=\left(D_{1}-\left|r_{1}\right|\right)\left(D_{2}-\left|r_{2}\right|\right)\left(D_{3}-\left|r_{3}\right|\right)$.

Notice that for fixed $s_{k}$ and $t_{k} s_{k}^{\prime} s_{1} s_{2} s_{3}^{\prime} s_{3} H_{t_{1} t_{2} t_{3}} \leftrightarrow H_{s s^{\prime} t}$ has value only when $s_{k}^{\prime}=s_{k}+t_{k}-2^{P_{k}}-1$ or $s_{k}^{\prime}=s_{k}+t_{k}-2^{P_{k}}$.

The final expression for the local OCF is obtained by incorporating (27) into (26)

$f_{2}\left(g, g^{\prime} \mid r\right) \approx H_{s s^{\prime} t} D_{s}^{n} D_{s^{\prime}}^{n^{\prime}} \chi^{n}(g) \chi^{n^{\prime}}\left(g^{\prime}\right) \chi_{t}(r)$.

Recalling relation (26), with Fourier coefficients of the local OCF, $F_{t}^{n n^{\prime}}$, and equating to relation (31), we obtain the fundamental relation in terms of the primitive Fourier coefficients:

$F_{t}^{n n^{\prime}}=H_{s s^{\prime} t} D_{s}^{n} D_{s^{\prime}}^{n^{\prime}}$

Thus, the coefficients of the local 2-point OCF, $F_{t}^{n n^{\prime}}$, are related through (32) to the coefficients of the specified sample microstructure, $D_{s}^{n}$. The latter represent coefficients of the local ODF specified in each cell of the sample as previously described. An analytical estimate for the weighting coefficients, $H_{s s^{\prime} t}$, is provided in Appendix A.

At this juncture an important observation can be made with reference to the fundamental relation (32). Approximations to the TF of resolution $1 / S$ in the spatial variables and resolution $1 / N$ in the orientation variables give rise to $N S$ independent Fourier coefficients, $D_{s}^{n}$. On the other hand, the same resolution in both types of variables requires $8 N^{2} S$ coefficients $F_{t}^{n n^{\prime}}$ of the OCF. Thus, relation (32) reflects the fact that only a small fraction of the $F_{t}^{n n^{\prime}}$ coefficients can be independent, since the true number of independent coefficients must be the same for the local OCF as it is for the sample TF. Indeed, the $r$-interdependence of the OCFs is massive, 
and must be dealt with in second-order microstructure design.

\section{The hull of texture functions}

The preceding development clarifies the relationship between OCFs and their TFs. Given the $r$-interdependence of the OCFs, it is preferable for the purposes of microstructure design to define a hull of texture functions, which is the set of all physically possible TFs. By definition, the TF for each element of the ensemble must belong to the hull of TFs. The question before us is how can this hull of TFs be constructed, and then explored to obtain all possible effective properties. Here, we take a particular approach that relates to our previous work on the hull of ODFs for the first-order microstructure design problem [6-9]. We first define the concept of eigen-TFs that comprise only single-orientation states (i.e., from only one sub-region of the FZ) in each sub-cell. Having fixed the spatial and orientational resolutions of the double-indicator primitive basis for TFs, we find that the hull of TFs is just the set of convex combinations of all possible eigen-TFs. Thus, the hull of TFs is a convex polyhedron in the $S N$ dimensional space of coefficients $D_{s}^{n}$.

\subsection{Eigen-texture functions}

Consider TFs that can be thought of as assigning a single lattice orientation $g_{n} \in \gamma_{n}$ to each sub-cell $\omega_{s}$. These are the eigen-TFs, and even for very ordinary resolutions in the spatial and orientational variables, they are very large in number. In fact, since there are $N$ possible orientations for each sub-cell $s$, there must be $N^{S}$ distinct eigen-TFs. Let them be enumerated by the index $j, 1 \leqslant j \leqslant N^{S}$. Use the symbol ${ }_{j} \hat{M}(x, g)$ to represent the $j$ th eigen-TF. Let the orientation index $n_{j}(s)$ identify the particular orientation that associates with sub-cell $s$ of the $j$ th eigen-TF. The Fourier coefficients for ${ }_{j} \hat{M}(x, g),{ }_{j} \hat{D}_{s}^{n}$, must have, according to (18), the form

${ }_{j} \hat{D}_{s}^{n}=N \delta_{n}^{n_{j}(s)}$.

Inputting (33) into (15) we obtain

${ }_{j} \hat{M}(x, g)={ }_{j} \hat{D}_{s}^{n} \chi_{s}(x) \chi^{n}(g)=N \sum_{s=1}^{S} \chi_{s}(x) \chi^{n_{j}(s)}(g)$.

It is evident that the Fourier coefficients of each eigenTF satisfy relations (17)-(19). Note that only NS of the $N^{S}$ eigen-TFs are linearly independent.

\subsection{Construction of the hull of texture functions}

Enumeration of the eigen-TFs can be accomplished in many ways. At this juncture we will not describe any particular method, but one approach is illustrated in Section 7 of the paper. Let the set of all eigen-TFs be $\hat{\mathbf{M}}$. From $\hat{\mathbf{M}}$ the hull of texture functions, $\mathbf{M}$, is readily constructed. Within a spatial resolution of $1 / S$ and an orientation resolution of $1 / N$, all possible TFs belong to the set of all possible convex combinations of the eigenTFs. Mathematically

$M(x, g)=\sum_{j=1}^{N^{S}}{ }_{j} \alpha_{j} \hat{M}(x, g)$

such that

$\sum_{j=1}^{N^{S}} \alpha=1,0 \leqslant{ }_{j} \alpha \leqslant 1$.

And $\mathbf{M}$ is defined as

$$
\begin{aligned}
\mathbf{M}=\{ & M(x, g) \mid M(x, g)=\sum_{j=1}^{N^{S}}{ }_{j} \alpha_{j} \hat{M}(x, g) ; \hat{M}(x, g) \in \hat{\mathbf{M}} ; \\
& \left.\sum_{j=1}^{N^{S}}{ }_{j} \alpha=1 ; 0 \leqslant{ }_{j} \alpha \leqslant 1\right\} .
\end{aligned}
$$

Associated with $\mathbf{M}$ is a related hull, comprising the convex hull of all possible Fourier coefficients associated with all TFs in M. Let this hull be $\tilde{\mathbf{M}}$. By combining (34) and (36) we have

$\tilde{\mathbf{M}}=\left\{D_{s}^{n} \mid D_{s}^{n}=\sum_{j=1}^{N_{j}}{ }_{j} \alpha_{j} \hat{D}_{s}^{n} ; \sum_{j=1}^{N_{j}} \alpha=1 ; 0 \leqslant{ }_{j} \alpha \leqslant 1\right\}$.

When combined with relation (33), expression (37) is amenable to the construction of the entire set of all possible Fourier coefficients belonging to all TFs.

Returning to the point that the set $\tilde{\mathbf{M}}$ contains eigenTFs that are not linearly independent of one another over the $S N$-dimensional Fourier space, we note that the set $\tilde{\mathbf{M}}$ does comprise the unique set from which all possible TFs can be constructed by convex combination. If a set of linearly independent eigen-TFs were selected from among $\tilde{\mathbf{M}}$, then all TFs can be formed by linear combination of this set. These combinations, however, will not be convex combinations. The advantage of forming TFs from $\tilde{\mathbf{M}}$ lies in the fact that convex combinations form a rule of mixtures for the formation of TFs, where any particular TF can be considered a mixture of eigen-TFs weighted by volume fractions that must sum to one.

\subsection{Geometrical interpretation of the hull of TFS}

A geometrical interpretation of $\tilde{\mathbf{M}}$ is readily achieved based on relation (37), subject to constraint relations (17)-(19). Eq. (37) delineates a hypercube of length $N$ 
(see relation (33)) in $S N$-dimensional Euclidean space. As required in (18), the sum of $D_{s}^{n}$ over all orientation sub-domains must equal $N$ for any choice of sub-cell $s$. Each such equation represents a hyper-plane in $D_{s}^{n}$-space. There are $S$ of these hyper-planes. The intersection of the $S$ constraining hyper-planes with the hypercube delineated by relation (37) then defines the convex hull $\tilde{\mathbf{M}}$. Further reflection reveals that the hull $\tilde{\mathbf{M}}$ is essentially a $S N-S$ dimensional polyhedron (including the surface and the interior), whose vertices are the distinct $N^{S}$ eigen-texture functions described earlier. ${ }^{1}$

Within the framework presented, it is also easy to isolate the sub-space of $\tilde{\mathbf{M}}$ that corresponds to a prescribed global ODF. The normalization relations $6,7,17,18$ require

$\sum_{s=1}^{S} D_{s}^{n}=V_{n} S N$

where $V_{n}$ denotes the volume fraction occupied by the orientations $g \in \gamma_{n}$. It is evident that constraint relation (38) is of similar kind as that implied by (17). There are $N-1$ constraint relations of type (38). Imposing these constraints on $\tilde{\mathbf{M}}$ identifies the subspace of $\tilde{\mathbf{M}}$, say $\tilde{\mathbf{M}} \mid V_{n}$, that is associated with a prescribed ODF. This subspace of $\tilde{\mathbf{M}} \mid V_{n}$ is an $(S-1)(N-1)$ dimensional polyhedron.

Arguably, the simplest example of a microstructure amenable to the geometrical interpretation just described is a two-dimensional microstructure occupying a square of size 1 . If we choose to partition this square into four sub-squares of equal size (length $1 / 2$ on each side), we have fixed $S=4$. Suppose that the local state space can be split into two sub-regions, each with measure $1 / 2$. Thus, $N=2$. The initial set of Fourier coefficients are thus $D_{1}^{1}, D_{1}^{2}, D_{2}^{1}, D_{2}^{2}, D_{3}^{1}, D_{3}^{2}, D_{4}^{1}, D_{4}^{2}$. Relation (19) indicates that each of these must satisfy the constraints $0 \leqslant D_{s}^{n} \leqslant 2$, and when combined with the four relations required by (18) (e.g. $\left.D_{1}^{1}+D_{1}^{2}=2\right)$, it is evident that all possible independent Fourier coefficients (say $D_{1}^{1}, D_{2}^{1}, D_{3}^{1}, D_{4}^{1}$ ) must lie in a 4-dimensional hypercube bounded by four sets of faces $D_{s}^{1}=0,2$. The points lying within or on the faces of this hypercube constitute the hull of TFs for this problem. This hypercube is also bounded by $N^{S}=16$ eigen-TFs, which are the corners of the polyhedron. These are the sets

$$
\left(D_{1}^{1}, D_{2}^{1}, D_{3}^{1}, D_{4}^{1}\right) \in\left\{\begin{array}{l}
(2,0,0,0),(0,2,0,0),(0,0,2,0),(0,0,0,2), \\
(2,2,0,0),(2,0,2,0),(2,0,0,2),(0,2,2,0), \\
(0,2,0,2),(0,0,2,2),(2,2,2,0),(2,2,0,2), \\
(2,0,2,2),(0,2,2,2),(2,2,2,2),(0,0,0,0)
\end{array}\right\} .
$$

\footnotetext{
${ }^{1}$ Another way to look at this comes from examining the number of independent Fourier coefficients. We begin with $S N$ of these, but add one constraint equation for each sub-cell. There are $S$ of these constraint equations, each of which can be used to remove one interdependent Fourier coefficient. Thus there are $S N-S$ independent coefficients forming a polytope residing in an $S N-S$ dimensional real space.
}

One way to visualize in 3-dimensions this 4-dimensional polyhedron is to add an additional constraint for volume fraction of phase $1, V_{1}=V$. Eq. (38) then has the form $D_{1}^{1}+D_{2}^{1}+D_{3}^{1}=8 V-D_{4}^{1}$. For fixed $V$, taking $D_{4}^{1}$ to lie in the range $0 \leqslant D_{4}^{1} \leqslant 2$ we see that $D_{1}^{1}, D_{2}^{1}, D_{3}^{1}$ must lie between the two bounding planes $D_{1}^{1}+D_{2}^{1}+D_{3}^{1}=8 \mathrm{~V}$ and $D_{1}^{1}+D_{2}^{1}+D_{3}^{1}=8 V-2$. The perpendicular distance between this pair of planes is always $2 / \sqrt{3}$. Given the fact that $0 \leqslant V \leqslant 1$, a continuous sequence of bounding planes is realized, spanning across the 3-dimensional cube in $D_{1}^{1}, D_{2}^{1}, D_{3}^{1}$. Their intersections comprise isolated points, tetrahedral of various sizes, and also 8-sided polyhedra of various sizes and shapes. The largest of these occurs when $V=1 / 2$. Figs. 1 and 2 depict these geometrical constructions.

\section{Transformations of coordinate basis}

The goal of this section is to transform the representation of the TF, with its primitive double-indicator basis, to an alternative basis consisting of products of generalized spherical harmonic functions (for the orientation variables) and Haar wavelets for the spatial variables. The chief advantage to representation of the orientation variables by generalized spherical harmonic functions is that this basis is known to be optimally efficient in describing functions that transform like tensors with coordinate change. The advantage with wavelet functions is that they are efficient in describing certain modulations of local state in space that are intuitive with respect to well known results of homogenization theory. Full description of the generalized spherical harmonic functions, symmetrized for the point symmetry subgroup of the pertinent crystal lattice, is beyond the scope of this paper. A full treatment, with many numerical helps, can be found in Bunge [10]. Likewise, the treatment of the Haar wavelet functions is limited; for deeper understanding textbook presentations can be consulted [20].

\subsection{Orientation symmetry in the texture functions}

The reader will notice a difference between the generalized spherical harmonic functions to be used hereafter and those used for representation of the ODF in relation (2). The ODF is typically constructed with basis functions of the type $\ddot{T}_{l}^{\mu v}(g) \leftrightarrow \dot{T}^{L}(g)$, which contain both crystallite and statistical (processing) symmetries. (This is signified by the set of three dots above the functions, and the use of the greek symbol $v$ for the third index, which is restricted to the range $1 \leqslant v \leqslant N(l)$, according to convention [10].) Basis functions used in representation of the TF would generally not satisfy the statistical (processing) symmetry condition, since this symmetry would normally be absent in the cubical sub-cells of 

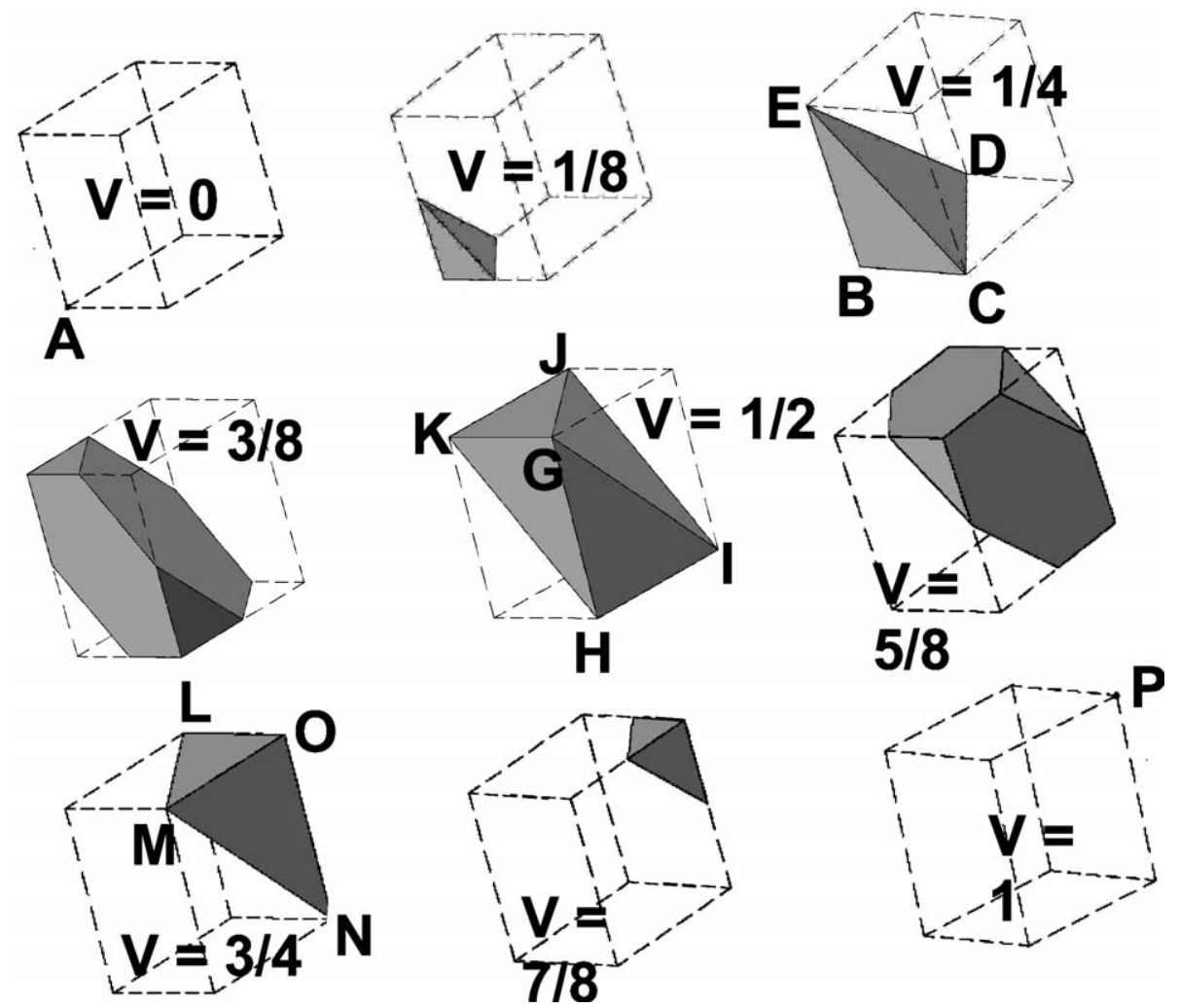

Fig. 1. Sequence of polytopes representing the hull of textures for $S=4, N=2$, with volume fractions $V$. (Coordinates are $D_{1}^{1}, D_{2}^{1}, D_{3}^{1}$. Vertices are coordinated with Fig. 2.)
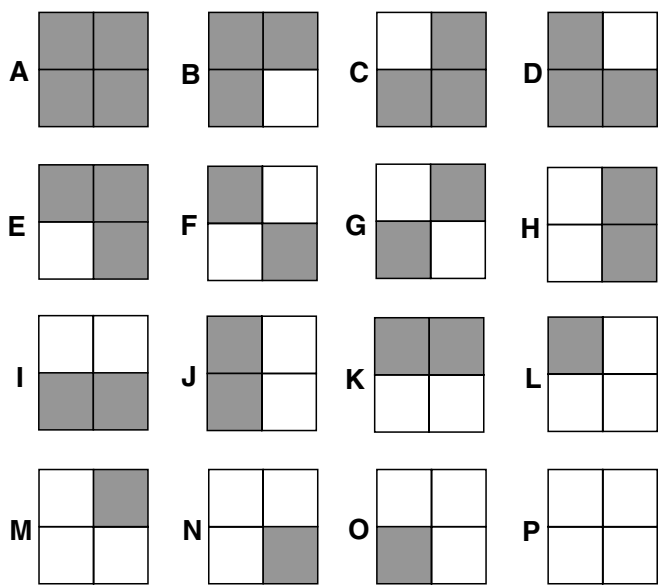

Fig. 2. Eigen-textures for $S=4, N=2$. (Labels correspond to vertices in Fig. 1.)

size $\delta$. (Hence, they are expressed in the form $\dot{T}_{l}^{\mu n}(g) \leftrightarrow$ $\dot{T}^{L}(g)$, with the two dots above the functions indicating crystallite symmetry only, and the use of index $n$, with $-l \leqslant n \leqslant+l$ as the third index, enumerating an expanded number of pertinent subspaces.)

\subsection{Haar wavelet functions}

Consider an alternative Fourier representation for the spatial component of the TF, involving elementary
Haar wavelet functions. We first present the equations in full (non-compacted) notation, and then transition to the compact form.

In the context of wavelets, $P_{k}$ denotes the generation of the Haar wavelet representation in $x_{k}$. Define the 1-dimensional father wavelet on the prescribed interval to be $W_{0}^{0}\left(x_{k}\right)$, where

$W_{0}^{0}\left(x_{k}\right)= \begin{cases}1 & \text { if } 0 \leqslant x_{k}<D_{k} \\ 0 & \text { otherwise }\end{cases}$

The reader should note the compact support of this function on the interval $\left[0, D_{k}\right)$.

The mother wavelet is defined to be

$W_{1}^{0}\left(x_{k}\right)= \begin{cases}+1 & \text { if } 0 \leqslant x_{k}<D_{k} / 2, \\ -1 & \text { if } D_{k} / 2 \leqslant x_{k}<D_{k}, \\ 0 & \text { otherwise. }\end{cases}$

Daughter wavelets are conveniently defined relative to the mother wavelets:

$W_{p}^{q}\left(x_{k}\right)=W_{1}^{0}\left(2^{(p-1)} x_{k}-q D_{k}\right)$
$\quad\left(p \geqslant 2 ; 0 \leqslant q \leqslant 2^{(p-1)}-1\right)$.

Utilizing the full set of Haar wavelets and the (symmetrized) generalized spherical harmonic functions, the alternative Fourier representation of $M(x, g)$ can readily be constructed: 


$$
\begin{aligned}
M(x, g) \approx & \sum_{p_{1}=0}^{P_{1}} \sum_{q_{1}=0}^{Q\left(p_{1}\right)} \sum_{p_{2}=0}^{P_{2}} \sum_{q_{2}=0}^{Q\left(p_{2}\right)} \sum_{p_{3}=0}^{P_{3}} \sum_{q_{3}=0}^{Q\left(p_{3}\right)} \sum_{l=1}^{L} \sum_{\mu=1}^{M(l)} \\
& \times \sum_{n=-l}^{+l} l_{\mu n} M_{p_{1} p_{2} p_{3}}^{q_{1} q_{2} q_{3}} W_{p_{1} p_{2} p_{3}}^{q_{1} q_{2} q_{3}}(x) \dot{T}_{l}^{\mu n}(g) .
\end{aligned}
$$

Here, the summation limits on the $q$-indices are

$Q(0)=Q(1)=0, \quad Q\left(p_{k} \geqslant 2\right)=2^{\left(p_{k}-1\right)}-1$.

The functions $W_{p_{1} p_{2} p_{3}}^{q_{1} q_{2} q_{3}}(x)$ in (42) are 3-dimensional Haar wavelet functions, comprising products of the 1-dimensional wavelets:

$$
W_{p_{1} p_{2} p_{3}}^{q_{1} q_{2} q_{3}}(x)=W_{p_{1}}^{q_{1}}\left(x_{1}\right) W_{p_{2}}^{q_{2}}\left(x_{2}\right) W_{p_{3}}^{q_{3}}\left(x_{3}\right) .
$$

These form a complete basis for functions in the 3 -dimensional real space that are piecewise continuous over the sub-cells $\omega_{s_{1} s_{2} s_{3}} \subset \Omega$.

Returning to the compact notational form, we set $W_{p_{1} p_{2} p_{3}}^{q_{1} q_{2} q_{3}}(x) \leftrightarrow W_{p}(x)$ and $\dot{T}_{l}^{\mu n}(g) \leftrightarrow \dot{T}^{L}(g)$. It follows that (42) accepts the form:

$M(x, g) \approx M_{p}^{L} W_{p}(x) \dot{\dot{T}}^{L}(g)$.

\subsection{Transformations between the two bases}

From the basic properties of the Haar wavelets and the primitive indicator functions, the following relationship exists between the two:

$\frac{1}{\delta^{3}} \iiint_{(i)_{\Omega}} W_{p}(x) \chi_{s}(x) \mathrm{d} x=\Pi_{p s} \in\{-1,0,+1\}$.

In words, the $\Pi_{p s}$ coefficients are integer-valued, and must be $-1,0$, or +1 .

In terms of the orientation variables we have the following approximation:

$\iiint_{\mathrm{FZ}} \chi^{n}(g) \dot{\dot{T}}^{L}(g) \mathrm{d} g \approx \dot{T}^{L}\left(g_{n}\right) / N$.

Recall that $g_{n}$ is the 'centroid' orientation of the subdomain $\gamma_{n}$.

Having defined the hull of TFs in the primitive double-indicator-function basis, where a relatively simple geometrical interpretation can be given, it is useful to consider the coordinate transformations that would enable $\tilde{\mathbf{M}}$ to be calculated for other coordinate bases. In particular it is useful to express the spatial dependence in terms of the Haar wavelet basis, and the orientation dependence in terms of the classical generalized spherical harmonic functions. The process of coordinate transformation proceeds readily from the following construction: Equate relations (15) and (45) for the TF. Multiply both sides by the basis product $\dot{T}^{L}(g) W_{p}(x)$ and integrate over the entire range of orien- tation and spatial variables. Since the generalized spherical harmonic functions have the property [10]

$$
\begin{aligned}
& \iiint_{\mathrm{FZ}} \dot{T}^{L}(g) \dot{T}^{* L^{\prime}}(g) \mathrm{d} g \\
& =\iiint_{\mathrm{FZ}} \dot{T}_{l}^{\mu n}(g) \dot{T}_{l^{\prime}}^{* \mu^{\prime} n^{\prime}}(g) \mathrm{d} g=\frac{\delta_{l}^{l^{\prime}} \delta_{\mu}^{\mu^{\prime}} \delta_{n}^{n^{\prime}}}{2 l+1} \leftrightarrow \frac{\delta_{L}^{L^{\prime}}}{2 l(L)+1},
\end{aligned}
$$

with $l(L)$ reminding us that we seek the index $l \in\{l, \mu, n\}$ associated with the compaction index $L$ on the right hand side. The Haar wavelets have the property

$$
\begin{aligned}
\iiint_{\Omega} W_{p^{\prime}}(x) W_{p}(x) \mathrm{d} x & =\iiint_{\Omega} W_{p_{1}^{\prime}}^{q_{1}^{\prime} q_{2}^{\prime} q_{3}^{\prime}}(x) W_{p_{1}}^{q_{1} q_{2} q_{3}}(x) \mathrm{d} x \\
& =\Lambda_{p_{1} p_{2} p_{3}}^{q_{1} q_{2} q_{3}} \delta_{p_{1}}^{p_{1}^{\prime}} p_{p_{2}}^{p_{2}} \delta_{p_{3}}^{p_{3}^{\prime}} \delta_{q_{1}}^{q_{1}^{\prime}} q_{q_{2}}^{q_{2}^{\prime}} \delta_{q_{3}}^{q_{3}^{\prime}} \leftrightarrow \Lambda_{p} \delta_{p}^{p^{\prime}},
\end{aligned}
$$

where the constants $\Lambda_{p}$ are real valued, and follow readily from the definition of the Haar wavelets (39)-(41) and (44), it follows that the side containing the TF expressed in wavelets and harmonics becomes, upon integration, $\frac{M_{p}^{L} \Lambda_{p}}{2 l(L)+1}$ (no summation over $p$ implied). The side containing the TF expressed in the double-indicator basis becomes

$D_{s}^{n} \iiint_{\Omega} \chi_{s}(x) W_{p}(x) \mathrm{d} x \iiint_{\mathrm{FZ}} \chi^{n}(g) \dot{\dot{T}}^{L}(g) \mathrm{d} g$.

Combining this with (46) and (47) we obtain

$M_{p}^{L}=\left[\frac{\Pi_{p s} \delta^{3}(2 l(L)+1) \dot{T}^{L}\left(g_{n}\right)}{N \Lambda_{p}}\right] D_{s}^{n}={ }_{p}{ }_{p} J_{s}^{n} D_{s}^{n}$.

With appropriate choices for truncation in each basis, the coefficients ${ }_{p}^{L} J_{s}^{n}$ can be chosen to form a $S N \times S N$ square matrix that is invertible. (This compatibility between the two bases, leading to the same dimensionality of the representations, and to invertibility, is required by the development that follows.) Let the inverse have coefficients ${ }_{s}^{n} J_{p}^{L}$, such that

$D_{s}^{n}={ }_{s}^{n} \breve{J}_{p}^{L} M_{p}^{L}$.

\subsection{Geometrical interpretation of the coordinate transformations}

Note that the coordinate transformation described in (51) and (52) is a linear one. Linear transformations transform lines into lines, planes into planes, etc. Thus, given that the hull of TFs in the primitive double-indicator space is a polyhedron, it must remain a polyhedron when transformed into the space of Haar wavelets and generalized spherical harmonic functions. 
5.5. The fundamental equation expressed in the new coordinate basis

Relation (52) is readily combined with the Fourier representation of the fundamental relation (32) to obtain the fundamental relation in the new coordinate basis:

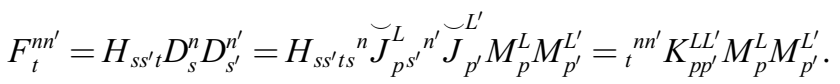

\section{Effective properties in the Fourier space}

Second-order estimates of effective properties require input of the 2-point OCFs. This is illustrated for the effective elastic stiffness tensor in Eq. (1) that contains a fourthorder tensor that can be written in index form as

$\left\langle C^{\prime} \Gamma C^{\prime}\right\rangle_{i j k l}=\iiint_{\Omega} \Gamma_{m n o p}^{r}\left(x-x^{\prime}\right)\left\langle C_{i j m n}^{\prime}(x) C_{o p k l}^{\prime}\left(x^{\prime}\right)\right\rangle \mathrm{d} x^{\prime}$.

Here, $\Gamma^{\mathrm{r}}\left(x-x^{\prime}\right)$ is the fourth-order Green's function tensor appropriate for the solution of the elastic field equations in a selected homogeneous reference medium subjected to homogeneous (displacement) boundary conditions [21]. For statistically homogeneous microstructures, the ensemble-averaged term in the integrand can be written as a function of $r=x^{\prime}-x$,

$$
\begin{aligned}
\left\langle C_{i j m n}^{\prime}(\vec{x}) C_{o p k l}^{\prime}\left(\vec{x}^{\prime}\right)\right\rangle= & \iiint_{\mathrm{FZ}} \iiint_{\mathrm{FZ}} f_{2}\left(g, g^{\prime} \mid r\right) \\
& \times\left(g \mathcal{C}^{\prime}\right)_{i j m n}\left(g^{\prime} \stackrel{\prime}{C}^{\prime}\right)_{o p k l} \mathrm{~d} g \mathrm{~d} g^{\prime},
\end{aligned}
$$

where

$$
(g \stackrel{\prime}{C})_{i j m n}=g_{i s} g_{j t} g_{m u} g_{n v}\left(\widehat{C}_{s t u v}-C_{s t u v}^{r}\right)
$$

is the polarization of the local stiffness tensor at orientation $g$, expressed in terms of $C$, the elastic stiffness tensor of the crystal in its reference orientation $g=I$. Incorporating (55), relation (54) can be rewritten as

$$
\begin{aligned}
& \left\langle C^{\prime} \Gamma C^{\prime}\right\rangle_{i j k l} \\
& =\iiint_{\Psi(\Omega)} \Gamma_{\text {mnop }}^{r}(-r) \iiint_{\mathrm{FZ}} \iiint_{\mathrm{FZ}} f_{2}\left(g, g^{\prime} \mid r\right) \\
& \quad \times\left(g \mathcal{C}^{\prime}\right)_{i j m n}\left(g^{\prime} C^{\prime}\right)_{o p k l} \mathrm{~d} g \mathrm{~d} g^{\prime} \mathrm{d} r .
\end{aligned}
$$

We introduce new notation for part of the integrand of Eq. (57),

$\Gamma_{\text {mnop }}^{r}(-r)\left(g \stackrel{\mathcal{C}^{\prime}}{)_{i j m n}\left(g / \stackrel{C}{C}^{\prime}\right.}\right)_{\text {opkl }}=\xi_{2(i j k l)}\left(g, g^{\prime}, r \mid \widehat{C}\right)$,

where $\left(g, g^{\prime}, r \mid \widehat{C}\right)$ indicates that $g, g^{\prime}$ and $r$ are the function variables, and $C$ is considered fixed. It is readily seen that the essential form of (57) is

$$
\begin{aligned}
& \left\langle C^{\prime} \Gamma C^{\prime}\right\rangle_{i j k l} \\
& =\iiint_{\Psi(\Omega)} \iiint_{\mathrm{FZ}} \iiint_{\mathrm{FZ}} f_{2}\left(g, g^{\prime} \mid r\right) \\
& \quad \times \xi_{2(i j k l)}\left(g, g^{\prime}, r \mid \widehat{C}\right) \mathrm{d} g \mathrm{~d} g^{\prime} \mathrm{d} r .
\end{aligned}
$$

It is notable that $\xi_{2(i j k l)}\left(g, g^{\prime}, r \mid \widehat{C}\right)$ effectively separates that portion of the integrand that carries the Green's function tensor, and the properties of the reference stiffness tensor. When the Green's functions themselves are selected such that they are independent of microstructure itself, then a convenient separable form of relation (59) is obtained. In the sequel it is considered that the Green's functions are separable in this manner. Forming a Fourier representation for $\xi_{2(i j k l)}\left(g, g^{\prime}, r \mid \widehat{C}\right)$,

$\left.\xi_{2(i j k l)}\left(g, g^{\prime}, r \mid \widehat{C}\right) \approx \Xi_{t}^{n n^{\prime}}{ }_{i j k l}, \widehat{C}\right) \chi^{n}(g) \chi^{n^{\prime}}\left(g^{\prime}\right) \chi_{t}(r)$,

and introducing it into (59), with reference to (11), (14) and (26), yields the expression

$\left\langle C^{\prime} \Gamma C^{\prime}\right\rangle_{i j k l}=\left\langle\widetilde{\Xi}_{t}^{n n^{\prime}}\left({ }_{i j k l}, \widehat{C}\right) F_{t}^{n n^{\prime}}\right\rangle$,

where the renormalized coefficients are

$\tilde{\Xi}_{t}^{n n^{\prime}}\left({ }_{i j k l}, \widehat{C}\right)=\left[\frac{\delta^{3}}{N^{2}}\right] \Xi_{t}^{n n^{\prime}}\left({ }_{i j k l}, \widehat{C}\right)$.

The notation for the Fourier coefficients, $\Xi_{t}^{n n^{\prime}}\left({ }_{i j k l}, \widehat{C}\right)$, reminds us that they depend upon the reference elastic constants and on the particular ijkl component that is being considered, but not upon any aspect of microstructure. Introducing (32) into (61) and separating out the component that is affected by ensemble averaging, we obtain

$$
\left\langle C^{\prime} \Gamma C^{\prime}\right\rangle_{i j k l}=\left\langle\tilde{\Xi}_{t}^{n n^{\prime}}\left(_{i j k l}, \widehat{C}\right) H_{s s^{\prime} t} D_{s}^{n} D_{s^{\prime}}^{n^{\prime}}\right\rangle=\aleph_{s s^{\prime}}^{n n^{\prime}}\left({ }_{i j k l}, \widehat{C}\right)\left\langle D_{s}^{n} D_{s^{\prime}}^{n^{\prime}}\right\rangle .
$$

If the classical coordinate frame is preferred, relation (53) is introduced into (61) to obtain

$$
\begin{aligned}
\left\langle C^{\prime} \Gamma C^{\prime}\right\rangle_{i j k l} & =\left\langle\tilde{\Xi}_{t}^{n n^{\prime}}\left({ }_{i j k l}, \widehat{C}\right)^{n n^{\prime}}{ }_{t} K_{p p^{\prime}}^{L L^{\prime}} M_{p}^{L} M_{p^{\prime}}^{L^{\prime}}\right\rangle \\
& =\aleph_{p p^{\prime}}{ }^{L L^{\prime}}\left({ }_{i j k l}, \widehat{C}\right)\left\langle M_{p}^{L} M_{p^{\prime}}^{L^{\prime}}\right\rangle .
\end{aligned}
$$

Thus, the second-order correction term in the effective property relation (1) is expressed in (63) and (64) as a sum over the $4 \leftrightarrow 12$ indices of the $\aleph$ (or $\tilde{\aleph}$ ) coefficients weighting the ensemble average of a particular quadratic product of the Fourier coefficients of the local OCF.

\subsection{Interpretation of the effective properties relationships in terms of the set of eigen-TFs}

The principal advantage of constructing the hull of TFs from the set of eigen-TFs can now be clearly demonstrated in terms of Eqs. (63), (64) for the second-order correction to the elastic effective properties. Recalling the meaning of ensemble averaging, as described in relation (21), relations (63), (64) can be rewritten as 


$$
\begin{aligned}
\left\langle C^{\prime} \Gamma C^{\prime}\right\rangle_{i j k l} & =\aleph_{s s^{\prime}}^{n n^{\prime}}\left({ }_{i j k l}, \widehat{C}\right)\left\langle D_{s}^{n} D_{s^{\prime}}^{n^{\prime}}\right\rangle \\
& =\left\langle\aleph_{s s}^{n n^{\prime}}\left({ }_{i j k l}, \widehat{C}\right) D_{s}^{n} D_{s^{\prime}}^{n^{\prime}}\right\rangle \\
& =\left\langle{ }^{(k)}\left(C^{\prime} \Gamma C^{\prime}\right)_{i j k l}\right\rangle
\end{aligned}
$$

and

$$
\begin{aligned}
\left\langle C^{\prime} \Gamma C^{\prime}\right\rangle_{i j k l} & =\aleph_{p p^{\prime}}^{\widetilde{L} L^{\prime}}\left({ }_{i j k l}, \widehat{C}\right)\left\langle M_{p}^{L} M_{p^{\prime}}^{L^{\prime}}\right\rangle \\
& =\left\langle\aleph_{p p^{\prime}}^{\leftarrow L^{\prime}}\left({ }_{i j k l}, \widehat{C}\right) M_{p}^{L} M_{p^{\prime}}^{L^{\prime}}\right\rangle \\
& =\left\langle{ }^{(k)}\left(C^{\prime} \Gamma C^{\prime}\right)_{i j k l}\right\rangle,
\end{aligned}
$$

where ${ }^{(k)}\left(C^{\prime} \Gamma C^{\prime}\right)_{i j k l}$ is interpreted to be the contribution to the property correction arising from the $k$ th element of the ensemble. Since all possible elements of the ensemble of TFs can be formed by convex combinations of the eigen-TFs belonging to $\tilde{\mathbf{M}}:^{2}$

$$
\begin{aligned}
& \left\langle D_{s}^{n} D_{s^{\prime}}^{n^{\prime}}\right\rangle \in\left\{\breve{C}_{D_{s} D_{s^{\prime}}^{n} D^{n^{\prime}}} \breve{D}_{s}^{n \smile D_{s^{\prime}}}=\sum_{j=1}^{N^{S}} \sum_{k=1}^{N^{S}}{ }_{j} \alpha_{k} \alpha_{j} \hat{D}_{s}^{n} \hat{D}_{s^{\prime}}^{n^{\prime}},\right. \\
& \left.\sum_{j=1}^{N^{S}}{ }_{j} \alpha=1,0 \leqslant{ }_{j} \alpha \leqslant 1,{ }_{j} \hat{D}_{s}^{n} \in \tilde{\mathbf{M}}\right\} \text {. }
\end{aligned}
$$

Similarly,

$$
\begin{aligned}
&\left\langle M_{p}^{L} M_{p^{\prime}}^{L^{\prime}}\right\rangle \in\left\{\breve{M}_{p}^{L} \breve{M}_{p^{\prime}}^{L^{\prime}} \mid \breve{M}_{p}^{L} \breve{M}_{p^{\prime}}^{L^{\prime}}=\sum_{j=1}^{N^{S}} \sum_{k=1}^{N^{S}}{ }_{j} \alpha_{k} \alpha_{j} \hat{M}_{p k}^{L} \hat{M}_{p^{\prime}}^{L^{\prime}},\right. \\
&\left.\sum_{j=1}^{N^{S}} \alpha=1,0 \leqslant{ }_{j} \alpha \leqslant 1,{ }_{j} \hat{M}_{p}^{L}={ }_{p}{ }_{p} J_{s j}^{n} \hat{D}_{s}^{n},{ }_{j} \hat{D}_{s}^{n} \in \tilde{\mathbf{M}}\right\} .
\end{aligned}
$$

\subsection{The second-order property correction closure}

Combining (65) with (67), and (66) with (68) we find that all possible second-order property corrections must be convex combinations of the quadratic products of eigen-TFs:

$$
\begin{aligned}
\left\langle C^{\prime}\right. & \left.\Gamma C^{\prime}\right\rangle_{i j k l} \\
= & \aleph_{s s^{\prime}}^{n n^{\prime}} \sum_{j=1}^{N^{S}} \sum_{k=1}^{N_{j}} \alpha_{k} \alpha_{j} \hat{D}_{s}^{n}{ }_{k} \hat{D}_{s^{\prime}}^{n^{\prime}} \\
& \left(\text { for } \sum_{j=1}^{N^{S}} \alpha=1,0 \leqslant{ }_{j} \alpha \leqslant 1,{ }_{j} \hat{D}_{s}^{n} \in \tilde{\mathbf{M}}\right) \\
= & \tilde{\aleph}_{p p^{\prime}}^{L L \prime} \sum_{j=1}^{N^{S}} \sum_{k=1}^{N^{S}}{ }_{j} \alpha_{k} \alpha_{j} \hat{M}_{s k}^{n} \hat{M}_{s \prime}^{n \prime} \\
& \left(\text { for } \sum_{j=1}^{N^{S}} \alpha=1,0 \leqslant{ }_{j} \alpha \leqslant 1,{ }_{j} \hat{M}_{p}^{L}={ }_{p}{ }_{p} J_{s j}^{n} \hat{D}_{s}{ }^{n},{ }_{j} \hat{D}_{s}^{n} \in \tilde{\mathbf{M}}\right) .
\end{aligned}
$$

\footnotetext{
${ }^{2}$ The reader will note that the condition $\sum_{j j} \alpha=1$ also implies that $\sum_{j} \sum_{k j} \alpha_{k} \alpha=1$. Thus, relations (67) and (68) can be considered as forming convex combinations among the quadratic products of the primitive coefficients of the eigen-texture functions.
}

Thus, all possible second-order property corrections can be constructed from convex combinations of quadratic products of the eigen-TFs. The set of all possible second-order property corrections is called the second-order property correction closure.

\section{Design of texture functions for elastic properties}

Second-order design for elastic properties proceeds naturally from relation (69). The key idea is to identify microstructure ensembles whose component eigen-TFs give rise to useful combinations of properties. Suppose, for example, that our principal interest lies with $\left\langle C^{\prime} \Gamma C^{\prime}\right\rangle_{3333}$ and $\left\langle C^{\prime} \Gamma C^{\prime}\right\rangle_{1313}$. Assume that first-order design led to an optimized set of volume fractions $\left\{V_{n} \mid n=1, \ldots, N\right\}$ giving rise to satisfactory first-order estimates of $C_{3333}^{*}, C_{1313}^{*}$ via $\left\langle C_{3333}\right\rangle,\left\langle C_{1313}\right\rangle$. Holding the $V_{n}$ constant we may then proceed to enumerate the set of eigen-TFs that belong to $\tilde{\mathbf{M}} \mid V_{n}$. Let this set be $\hat{\mathbf{M}} \mid V_{n}$. For each ${ }_{j} \hat{D}_{s}^{n} \in \hat{\mathbf{M}} \mid V_{n} \quad$ (or $\quad{ }_{j} \hat{M}_{p}^{L}={ }_{p}{ }^{L} J_{s j}^{n} \hat{D}_{s}^{n}$ $\left.\left({ }_{j} \hat{D}_{s}^{n} \in \hat{\mathbf{M}} \mid V_{n}\right)\right)$ the contribution of the eigen-TF to the properties, say ${ }_{j}\left(C^{\prime} \Gamma C^{\prime}\right)_{3333},{ }_{j}\left(C^{\prime} \Gamma C^{\prime}\right)_{1313}$, can be computed, given that fixing the volume fractions of components of texture renders the $\aleph_{s s^{\prime}}^{n n n^{\prime}}$ coefficients independent of microstructure, as discussed in Section 6. The 2-dimensional properties closure for $\left\langle\mathrm{C}^{\prime} \Gamma \mathrm{C}^{\prime}\right\rangle_{3333}$ and $\left\langle C^{\prime} \Gamma C^{\prime}\right\rangle_{1313}$ is just the convex hull of all points with coordinates ${ }_{j}\left(C^{\prime} \Gamma C^{\prime}\right)_{3333},{ }_{j}\left(C^{\prime} \Gamma C^{\prime}\right)_{1313}$.

\subsection{The large numbers challenge}

The main challenge in forming the closure is determination of the elements of set $\hat{\mathbf{M}} \mid V_{n}$. The number of elements of $\hat{\mathbf{M}} \mid V_{n}, \mathrm{O}\left(\hat{\mathbf{M}} \mid V_{n}\right)$, equals

$\mathrm{O}\left(\hat{\mathbf{M}} \mid V_{n}\right)=\frac{S !}{\prod_{n=1}^{N} N_{n} !}$,

where $N_{n}$ denotes the number of sub-cells within region $\Omega$ that associate with sub-region $\gamma_{n}$ of the FZ. Even for relatively small models, with relatively coarse resolutions in spatial and orientational variables, $\mathrm{O}\left(\hat{\mathbf{M}} \mid V_{n}\right)$ can be very large. For example, consider a model with only $5 \times 5 \times 5=125$ sub-cells, and only 2 distinct orientation sub-regions. Imagine that 100 of the sub-cells associate with sub-region $\gamma_{1}$, and 25 with sub-region $\gamma_{2}$. For this case $\mathrm{O}\left(\hat{\mathbf{M}} \mid V_{n}\right) \approx 1.3 \times 10^{26}$. This large numbers challenge is central to second-order microstructure design. Sampling the set $\hat{\mathbf{M}} \mid V_{n}$ offers one approach for dealing with this problem. This approach has been taken in the case study.

\subsection{Case study in second-order elastic design}

We illustrate the basic concepts of second-order elastic design based upon $\hat{\mathbf{M}} \mid V_{n}$ for a microstructure 
Table 1

List of components of optimized ODF

\begin{tabular}{lllll}
\hline Component & $\varphi_{1}$ & $\phi$ & $\varphi_{2}$ & $V_{n}$ \\
\hline 0 & 3.51 & 0.95 & 0.58 & 0.49 \\
1 & 1.38 & 1.07 & 0.38 & 0.32 \\
2 & 4.95 & 1.28 & 0.28 & 0.19 \\
\hline
\end{tabular}

$\varphi_{1}, \phi, \varphi_{2}$ are the Euler angles of the orientations in the Bunge convention [10]. $V_{n}$ are the volume fractions.

previously obtained by first-order design over the hull of ODFs. The problem was to design an ODF that is predicted to maximize the (uniaxial) load bearing capacity of a plate containing a small hole. The problem was conducted for single-phase $\mathrm{Cu}$ polycrystals, restricted to the cubic-orthorhombic ODFs. Globally optimized Fourier coefficients were reported in previous publication [9]. The reported optimal texture, to a good approximation, can be approximated by the four orthorhombic variants of three single crystal components. The components and their volume fractions are listed in Table 1.

Restricting our calculations to effective elastic stiffnesses of the form $C_{i i i i}^{*}$ or $C_{i j i j}^{*}$ (summation not intended) enables the fundamental zone to be restricted to three elements $(N=3){ }^{3}$ The three components were distributed within a $4 \times 4 \times 4$ rectangular model $(S=64)$. Component 0 was distributed among 31 of the sub-cells, component 1 among 21, and component 2 among 12 . Approximately $3 \times 10^{6}$ eigen-texture functions were created by pair-wise switching of components among the sub-cells.

Elastic properties estimates were calculated using Eq. (1), with the second-order correction term evaluated using relation (69). The required $\aleph_{s s^{\prime}}^{n n^{\prime}}$ coefficients were calculated on the rectangular model based upon the isotropic Green's function tensor given by Kröner [21]. Ordinarily, given the natural spherical geometry of the problem, evaluation of the Green's functions may be conducted in expanding spherical shells in $r$-space. It is typically assumed that pair correlations beyond a coherence length for the microstructure, $r_{c}$, the 2-point OCFs decouple according to the expression $f_{2}\left(g, g^{\prime} \mid r>r_{c}\right)=f(g) f\left(g^{\prime}\right)$. The Green's functions of Kröner [21] no longer contribute to the effective properties beyond this coherence length. Here, however, spatial correlations are considered in a cubical region, rather than in a sphere, and hence a correction term is needed. Following the notation of Kröner, the second-order correction to the elastic properties is

\footnotetext{
${ }^{3}$ Each of the orthorhombic variants of the components has the same "diagonal" elastic stiffnesses; hence only one of the orthorhombic variants needs to be considered in the present calculations.
}

$$
\begin{aligned}
& \left\langle C^{\prime} \Gamma C^{\prime}\right\rangle_{i j k l} \\
& =\iiint_{\Psi(\Omega)} \Gamma_{n p q r}(r)\left\langle C_{i j n p}^{\prime}(x) C_{q r k l}^{\prime}(x+r)\right\rangle \mathrm{d} r \\
& \quad+\left\langle C_{i j n p}^{\prime}(x)\right\rangle\left\langle C_{q r k l}^{\prime}(x+r)\right\rangle \iiint_{\Sigma(\Omega)-\Psi(\Omega)} \Gamma_{n p q r}(r) \mathrm{d} r,
\end{aligned}
$$

where $\Sigma(\Omega)$ denotes the smallest sphere that contains the rectangular region $\Psi(\Omega)$. Thus, the region of integration for the left-most term in (71) is over the set of six caps that lie in the region of difference between $\Sigma(\Omega)$ and $\Psi(\Omega)$, or $\Sigma(\Omega)-\Psi(\Omega)$. In this region it is assumed that the microstructure is statistically uncorrelated, and hence the decoupling of the pair correlation functions is appropriate. Also, the reader will note that $\left\langle C_{i j n p}^{\prime}(x)\right\rangle$ and $\left\langle C_{q r k l}^{\prime}(x+r)\right\rangle$ in statistically homogeneous microstructures (as is the assumption here) are independent of position $x$ and $r$.

Basic elastic constants for the cubic copper crystal were taken to be, in units of GPa: $C_{11}^{o}=168.4$, $C_{12}^{o}=121.2$, and $C_{44}^{o}=75.4$. A Green's function was used, based upon an isotropic reference medium exhibiting Lame's constants calculated from the following ensemble averages:

$$
\begin{aligned}
& \mu_{\text {iso }}=\frac{1}{3}\left(\left\langle C_{1212}\right\rangle+\left\langle C_{3131}\right\rangle+\left\langle C_{2323}\right\rangle\right), \\
& \lambda_{\text {iso }}=\frac{1}{3}\left(\left\langle C_{1111}\right\rangle+\left\langle C_{2222}\right\rangle+\left\langle C_{3333}\right\rangle\right)-2 \mu .
\end{aligned}
$$

The results are shown in Figs. 3 and 4. Only a small fraction of the calculated points are shown in Fig. 3. We remark that the first-order Hill-Paul bounds are quite narrow for the optimized ODF, but the vast majority of calculations for estimating the second-order proper-

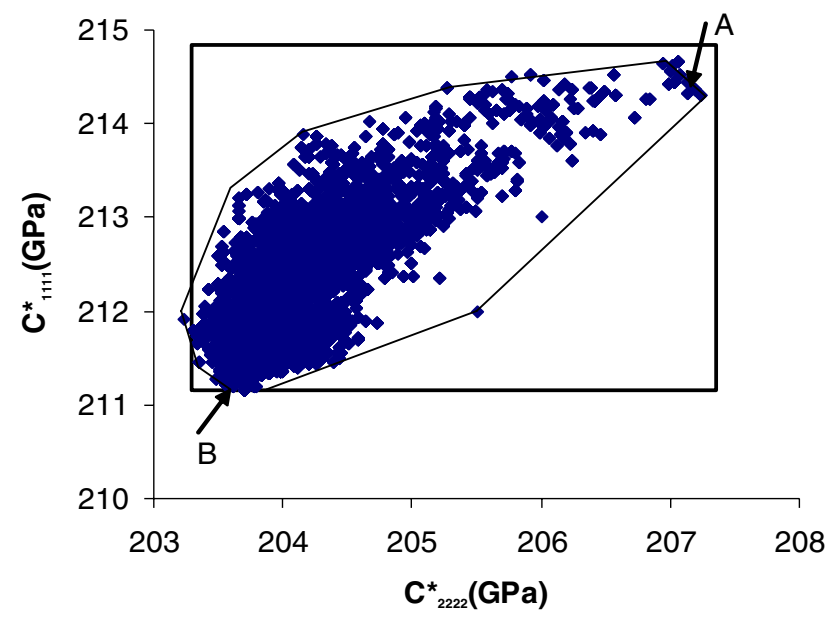

Fig. 3. Second-order combined elastic properties closure. (The black rectangular boundary comprises the Hill-Paul first-order bounds. Points indicate contributions of individual eigen-textures. The convex boundary indicates the approximate location of the boundary of the second-order (convex) properties closure. Points A and B correspond to configurations described in Fig. 4.) 

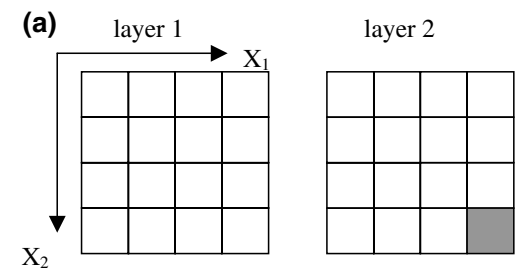

layer 3

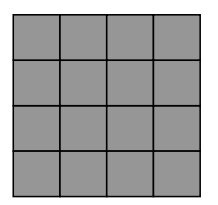

layer 3

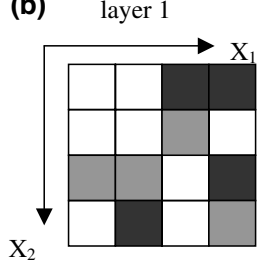

layer 2

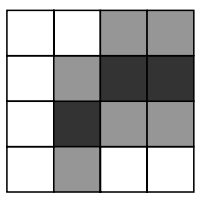

layer 4

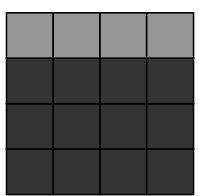

layer 4

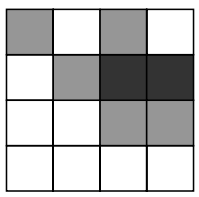

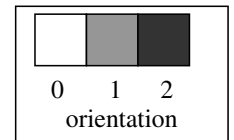

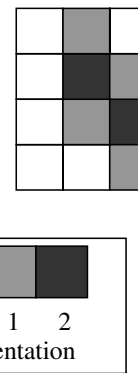

Fig. 4. Examples of specific configurations (eigen-textures) associated with extreme locations in the second-order elastic closure. (The shading scheme is correlated with Table 1.)

ties combinations lie within the first-order bounds, although a small number of cases were found that fall slightly outside. This is not surprising given the various numerical approximations used in obtaining the secondorder estimates.

\section{Summary and conclusions}

This paper shows how the challenging problem of $r$-interdependence of the 2-point OCFs can be overcome, by introducing the texture function as an intermediate construct. Texture functions, $M(x, g)$, describe the local distribution of lattice orientation $g$ at position $x$. A fundamental relation is given that links the 2-point OCFs, $f_{2}\left(g, g^{\prime} \mid r\right)$, to quadratic products of the texture functions.

Rectangular models were introduced to approximate the spatial dependence of the texture functions. Piecewise linear indicator functions were introduced as a primitive basis for both orientational and spatial variables on the domain of the texture function. These approximations give rise to a finite hull of texture functions, wherein all physically possible microstructures reside. The hull of texture functions is shown to be a convex polytope (hyper-cube) of dimension $S N$, where $S$ is the number of sub-cells in the rectangular model and $N$ is the number of sub-domains chosen to represent the fundamental zone of orientations. The corner (extreme) points of the hull (polytope) are found to number $N^{S}$. These corner states are called eigen-textures since they comprise cells that carry a single sub-domain of the fundamental zone.

Transformations of the hull of texture functions to other basis functions, viz. generalized spherical harmon- ics (orientational variables) and Haar wavelets (spatial variables) is approximated by a linear transformation law that leaves the basic geometry of the hull unchanged.

The 2-point OCFs, when introduced into appropriate homogenization relationships for effective properties, describe a family of generalized quadratic surfaces that intersect the hull of texture functions. All texture functions lying on any specified quadratic surface are predicted to be equipollent in terms of the specified effective property.

Second-order design of microstructure for specified properties combinations and constraints can proceed directly from consideration of the eigen-textures, in cases where the Green's functions are independent of microstructure. Combined properties combinations must lie in the convex combination of properties associated with component eigen-textures. The method is illustrated for elastic properties, beginning with an optimized ODF obtained by first-order texture design. Second-order estimates of the $C_{1111}^{*}$ vs $C_{2222}^{*}$ elastic closure were obtained for an ODF that was first optimized for (uniaxial) load bearing capacity in a plate containing a small hole, using the methods of first-order texture design [9]. These estimates were obtained using isotropic Green's functions, and methods that have been widely reported in the literature [1,13-15,21]. Recent results for obtaining Green's functions applicable to cubic polycrystals possessing orthorhombic symmetry, could potentially improve the results obtained for the case study reported here [22]. These estimates are found to be compatible with the first-order (Hill-Paul) bounds, and particular eigen-textures were found that are predicted to reach relative extremes in the closure. Thus, a limited invertibility of the second-order microstructure design methodology is demonstrated. 
The texture community is accustomed to considerations of statistical symmetry in the ODF itself, but the extent to which these symmetries must be observed spatially, in selected volume elements, is generally unknown. For the case study in this paper, the question is whether the orthorhombic symmetry can be, or must be present in the spatial distribution of the orientation components in a single $4 \times 4 \times 4$ model of the microstructure. This is not possible without violating the volume fraction condition. The reader should note that for the two elastic properties considered, $C_{1111}^{*}$ and $C_{2222}^{*}$, both are invariant with respect to the twofold orthorhombic symmetry elements. Thus, an orthorhombic cluster could be constructed from the derived $4 \times 4 \times 4$ model, which would possess orthorhombic symmetry, and which would have, to first-order, the same properties as the small model. Some minor differences in properties would accrue, primarily due to additional correlations (mostly of longer length scale) between sections in the larger model.

The main challenge to be addressed in applications is likely to be associated with the large numbers of eigen-textures comprising the set of extreme points of the polytope. It is anticipated that information about the sensitivities of properties to various components of the Fourier representation will need to be considered to optimize the search within this very large set.

\section{Acknowledgement}

This work was supported at Brigham Young University and Drexel University by a grant from the US Army Research Office, through the Metallurgy Program headed by Dr. David Stepp.

\section{Appendix A. Evaluation of $\boldsymbol{H}_{s s^{\prime} t}$}

The values of the components of $H_{s s^{\prime} t}$ are determined by substituting relation (30) into relation (29) and evaluating the integral. As noted earlier, this integral produces non-zero values only when $s_{k}^{\prime}=s_{k}+$ $t_{k}-2^{P_{k}}-1$ or $s_{k}^{\prime}=s_{k}+t_{k}-2^{P_{k}}$. Furthermore, in evaluating this integral, one needs to pay attention to the sign of each of the components of the variable $r$. It can be shown that

$$
\begin{aligned}
H_{s s^{\prime} t} & \leftrightarrow s_{1}^{\prime} s_{1}^{\prime} s_{2}^{\prime} s_{s_{2}} s_{3} H_{t_{1} t_{2} t_{3}} \\
& \approx h\left(s_{1}, s_{1}^{\prime}, t_{1} \mid P_{1}\right) h\left(s_{2}, s_{2}^{\prime}, t_{2} \mid P_{2}\right) h\left(s_{3}, s_{3}^{\prime}, t_{3} \mid P_{3}\right),
\end{aligned}
$$

where

$$
h\left(s, s^{\prime}, t \mid P\right)=\left\{\begin{array}{c}
-1-\left(2^{P+1}-t+1\right) \ln \left(\frac{2^{P+1}-t}{2^{P+1}-t+1}\right) \\
\quad \text { if } t=s^{\prime}-s+2^{P} \text { and } s^{\prime}>s \\
\left.1+2^{P+1}-t\right) \ln \left(\frac{2^{P+1}-t}{2^{P+1}-t+1}\right) \\
\quad \text { if } t=s^{\prime}-s+2^{P}+1 \text { and } s^{\prime} \geqslant s \\
1-(t-1) \ln \left(\frac{t}{t-1}\right) \\
\text { if } t=s^{\prime}-s+2^{P} \text { and } s^{\prime} \leqslant s \\
-1+t \ln \left(\frac{t}{t-1}\right) \\
\text { if } t=s^{\prime}-s+2^{P}+1 \text { and } s^{\prime}<s \\
0 \quad \\
\text { if } t \neq s^{\prime}-s+2^{P} \text { and } \\
t \neq s^{\prime}-s+2^{P}+1 .
\end{array}\right.
$$

Note that the relations (A.2) possess a singularity at the edges of the $\Psi(\Omega)$, when $t=1$ or $t=2^{P+1}$. Furthermore, in most cases, one might find the following approximation of relation (A.2) very useful.

$$
h\left(s, s^{\prime}, t \mid P\right)=\left\{\begin{array}{l}
\frac{1}{2\left(2^{P}-\left|t-2^{P}-\frac{1}{2}\right|\right)} \\
\text { if } t=s^{\prime}-s+2^{P} \quad \text { or } t=s^{\prime}-s+2^{P}+1, \\
0 \\
\text { if } t \neq s^{\prime}-s+2^{P} \text { and } t \neq s^{\prime}-s+2^{P}+1 .
\end{array}\right.
$$

\section{References}

[1] Kröner E. J Mech Phys Solids 1977;25:137.

[2] Zeller R, Dederichs PH. Phys Stat Solidi (B) 1973;55:831.

[3] Beran M, Molyneaux JQ. Appl Math 1966;24:107.

[4] Molinari A, Canova GR, Ahzi S. Acta Metall 1987;35:2983.

[5] Adams BL, Field DP. Acta Metall Mater 1991;39:2405.

[6] Adams BL, Henrie A, Henrie B, Lyon M, Kalidindi SR, Garmestani H. J Mech Phys Solids 2001;49:1639.

[7] Adams BL, Lyon M, Henrie B. Int J Plasticity 2004;20:1577.

[8] Kalidindi SR, Houskamp J, Lyon M, Adams BL. Int J Plasticity 2004;20:1561.

[9] Lyon M, Adams BL. J Mech Phys Solids 2004;52:2569.

[10] Bunge H-J. Texture analysis in materials science. London: Butterworths; 1982.

[11] Milton GW, Cherkaev AV. J Eng Mater Tech 1995;117:483.

[12] Lurie KA, Cherkaev AV. J Optim Th Appl 1984;42:283.

[13] Mason TA, Adams BL. Metall Mater Trans A 1999;30:969.

[14] Torquato S. Random heterogeneous materials. New York (NY): Springer; 2002.

[15] Morawiec A. Orientations and rotations. Berlin: Springer-Verlag; 2004.

[16] Bunge H-J, Schwarzer RA. Adv Eng Mater 2001;3:25.

[17] Man C-S. Arch Rational Mech Anal 1998;143:77.

[18] Schmidt S, Huang X, Juul-Jensen D. Mater Sci Forum 2004;467470:189.

[19] Spowart JE, Mullens HM, Puchala BT. J Metals 2003. October.

[20] Aboufadel E, Schlicker S. Discovering wavelets. New York (NY): John Wiley; 1999.

[21] Kröner E. In: Gittus J, Zarka J, editors. Modeling small deformations of polycrystals. London: Elsevier Applied Science; 1986.

[22] Huang. J Elast 2003;73:1. 